\title{
Bio-Informatics - Review on Current Research and Evolution of Cancer Targeted Therapies
}

\author{
Ayan Chatterjee \\ Department of IT, Jadavpur \\ University \\ Kolkata, India
}

\author{
Uttam Kumar Roy, PhD \\ Department of IT, Jadavpur \\ University \\ Kolkata, India
}

\author{
Nibedita Pahari \\ Computer Sc. \& Engineering, \\ WBUT \\ Kolkata, India
}

\begin{abstract}
The statistics shows that 7 million people die each year worldwide from cancer related cases \& by 2020 this number will be more than around 16 million as methods of cancer treatment \& cancer classification have become most challenging. So, cancer has become a noted disease responsible for major death rate. 200 different types of cancers are identified but some of them like lung, liver, stomach, colorectal, breast, oesophagal are leading death count. More than $60 \%$ of world's total new cases occur in Africa, Asia and Central and South America per annum. These regions account for $70 \%$ of the world's cancer deaths by oesophagal cancers as reported by World Health Organization.
\end{abstract}

This article reviews and discusses recent improvement in the treatment of cancer and the challenges that exist, in reduced cost \& time. Development and use of bioinformatics, nanotechnology is essential for the future cancer therapeutics. Most cancer treatments work for limited number of patients and this is likely to remain true for many molecularly-targeted drugs. As a consequence, a large proportion of patients are deprived of effective treatments and it is a huge financial burden on our health care system. It is essential to develop accurate tools for delivering the right treatment to the right patient based on biological characterization of each patient's tumor. The integration of wet experiments and the use of bioinformatics analysis have become an important part of biological and clinical research of this century. If we don't pay attention to it in future we will find a cancer patient in each family across the world. Cancer cure is really difficult but not complete hopeless. A lot of researches are being carried out in the field of bio-informatics to open up new possibilities for cancer treatments.

\section{Keywords}

Ligand; Microarray; Target; PDB; cell cycle; Oncogenes; Tumor; Peptide; DNA/Gene; Tumor Suppressor; Kinase etc.

\section{INTRODUCTION}

Cancer is typified as genetic mutation that forces to lose affected cells restricted division leading to formation of malignant tumor, error in protein formation \& metastasis. Angiogenesis is the most important \& fundamental step in transition of tumor from dormant to malignant state. Cancer cells originate from normal healthy cells and go on uncontrolled cell division after losing normal cell properties. They can adversely affect the healthy cells that frequently divide in the body - bone marrow.

First description of cancer was found in an Egyptian papyrus around 1600 BC. Surgery was most preferred way of treatment until 1950. After 1960, radiation therapy was being used to control local disease. However, all such treatments were not effective for metastasis cancer as therapy needed to reach every organ of the body. Therefore, current efforts to cure cancer are focusing on drugs, biological molecules and immune mediated therapies. Potential to fight this disease were intensified when the National Cancer Act was published in 1971 and President Nixon declared a war against cancer.

Till now cancer treatments are based on chemotherapy \& radiation are neither target specific or able to carry correct amount of drug to cancer cells thereby affect neighbor cells. Drug resistance, high fluid pressure, altered bio-distribution, bio-transformation \& drug clearance also create problems in traditional cancer treatments.

Targeted therapy provides us a wide variety of direct and indirect approaches. In these approaches, drugs can actively target tumor using tumor-specific MoAbs or peptide ligands binding to receptors that are present on tumor cells. With the active targeting, tumors can also be passively targeted by macromolecules. Discovery of protein or peptide receptors \& tumor related proteins has opened a new door of modern cancer treatments.

\section{CANCER CELLS}

\subsection{Cell Cycle, Gene \& Cancer}

Cancer is not a single disease. As per the medical reports, more than 200 types of cancers have been identified but lung, breast, colon, prostate and blood cancers are reported more than $50 \%$ globally. It is a cell cycle disease in which all regulatory controls go away leading to extra mass of tissue formation - called tumor. Tumors are found all kind of tissues and can be benign (Papilloma, Adenoma, Lipoma, Osteoma, Myoma, Angioma, Nevus) or malignant (Carcinomas- skin, mouth, lung, breast, stomach, colon, uterus \& Sarcomaslung). Benign tumors are not cancer but malignant tumors are cancer and they accelerate further cell division in unrestrained manner.

Cancer cell looses basic cell properties like - restricted cell reproduction, cell communication (signal of protein formation), cell adhesion (can move any part of the body through bloodstream), cell specialization \& cell death. Uncontrolled growth of the tumor cell is due to derailment of the cell cycle and its checkpoints, caused by mutation (any change of DNA molecule at molecular level due to chromosome replication error results in error protein synthesis) so that pathways for excessive propagation are activated while pathways that keep a check on cell cycle are silenced.

In humans, 287 genes out of around 30,000 (with $80 \%$ redundancy) are known to be disease genes. Genetic modification disrupts the encoding of cellular signaling molecules especially protein kinases, can results in cancers. 
Many types of gene alterations can occur in cancers \& four main types include:

- SNVs/Single nucleotide variants or point mutations: SNVs result from a base substitution at one nucleotide. a single nucleotide change results in a codon that codes for a different amino acid \& results in non functional protein product (missense mutation) or a premature stop codon or nonsense codon in the transcribed m-RNA \& results in truncated, incomplete, non functional protein product (non-sense mutation).

- Frame shift mutation or framing error: It changes the codon grouping and results in different translation from original and hence more altered protein gets formed.

- Gene copy number changes: They are caused by structural rearrangement of genome like large duplications, deletions, inversions or translocations encompassing entire exons and affecting the functional domains of the protein. This variation accounts for roughly $12 \%$ of human genomic DNA and each variation may range from about one kilo base $(1,000$ nucleotide bases) to several mega bases in size.

- A Structural variants (SVs) or large structural glitches of genetic material including translocations or inversions: often result in fusion genes and associated fusion proteins. [1].

Mutations happening in proto-oncogenes and tumor suppressor genes induce the cells to defy tight controls exerted in the cell division cycle. A glaring abnormality of the checkpoint in G1 phase has been observed. In certain tumors, the $\mathrm{Rb}$ gene is mutated, preventing the binding of $\mathrm{Rb}$ with E2F. Deregulation of Rb/E2F pathway is the feature of tumor cells caused by abnormal $\mathrm{Rb}$ control [1, 4].

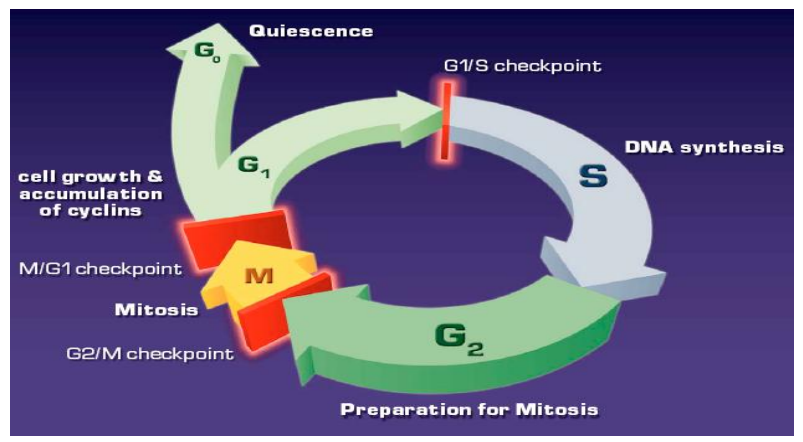

Fig 1: Cell Cycle \& Cancer

\subsection{Existing Therapies \& New Ideas}

Cancer treatment is a great challenge to medical professionals as it is very tough to distinguish between normal cells and cancer affected cells and target them.

Some cancers, particularly leukemia, are treated with very high doses of chemotherapy drugs or radiation with an intension to kill all responsible the cancer cells. The side effect of this harsh treatment is destruction of the bone marrow that contains stem cells. Stem cells, immature cells that develop into blood cells, are essential. So, after treating, patient's bone marrow must be restored, either from bone marrow removed from the patient before drug therapy or from a compatible/correct donor. Although the patient's own bone marrow is the best, but it can contain cancer cells so that must be destroyed before it is returned back to the patient. Chemotherapy drugs also kill certain adult cells that divide faster, and affect gastrointestinal tract, bone marrow cells, and hair follicles and causes some of the side effects including gastrointestinal distress, low white blood cell count, and hair loss.

Pharmacogenetics can be applied to the cases in which individuals have dihydropyrimidine dehydrogenase/DPD deficiency. The patients with this rare genetic disorder develop neurotoxicity when treated with common chemotherapy such as 5-flurouracil.

Drugs prescribed for cancer are cytotoxic in nature. Traditional radiation and chemotherapeutic treatments have a limitation over discriminating normal \& abnormal cells, hence are unable to target abnormalities associated with malignant tumor cells. These therapies, directly or indirectly, act on the cells cycle causing disturbance of the cyclic events, which are otherwise precisely controlled.

A class of drugs called spindle inhibitors (stop the synthesis of microtubules) stops cell replication early in mitosis. Experts have suggested that in order to overcome the side effects, efforts should be aimed at finding new and efficacious anticancer drugs based on identification of the cell cycle targets that interfere with the cell cycle regulatory pathway.

A very few cell cycle targets have been exploited for designing anticancer drugs, the identification \& validation of more targets holds promise in selective cancer therapy. Drosophila Melanogaster is one of the model organisms that have been used for the purpose. The link between the cell cycle \& chromatin control has led to untie the mechanisms to explain abnormalities of tumor cells that can be targeted to design new drugs. Targeting chromatin control may yield valuable information.

Since cancers originate from abnormalities in DNA sequence, a comparison in between normal and tumor cells may reveal therapeutic targets. Some cancers are, however, caused by a single gene abnormality, while most are heterogeneous \& multigenic. If mutant genes can be identified, they could prove to be effective targets. Prime growth factor of normal cell is hormones. Although cancer cells have lost some of the normal responses to growth factors, but some cancer cells require hormones to grow up. Hormone therapy for cancer attempts to starve the cancer cells of these hormones. It is usually done with drugs that block the activity of the hormone, although some drugs can block synthesis of the hormone. Drugs block the binding site for hormone to slow the growth of these cancers. Research proves that it is effective for prostate $\&$ breast cancer.

Newer chemotherapeutic drugs target specific, active proteins or processes in cancer cell signal transduction pathways like receptors, growth factors, or kinase as the targets are cancerspecific proteins \& the hope is that these drugs will be much less toxic to normal cells than conventional cancer drugs.

Some drugs binds to abnormal proteins in cancer cells, blocking their action in promoting uncontrolled cell growth. Because it binds only to these abnormal proteins, they don't show the high levels of toxicity like other chemotherapy drugs. The oncogene namely RAS is mutated in many types of cancer, particularly pancreatic cancer. The RAS protein is only active after it is being modified by the addition of a specific chemical group. Scientists are developing drugs to inhibit the action of enzyme that adds the chemical group to RAS protein to make inactive form of RAS. Early tests indicate that such drugs show hope for reducing tumors in cancer patients. 
Chemotherapy may fail as the cancer cells can become resistant to the traditional therapeutic drugs. Cancer cells show a high frequency of mutation. In the presence of toxic drugs, cancer cells that mutate and become resistant to the drug will survive and divide in the presence of drug, resulting a tumor that is also resistant to the drug. To avoid this problem, combinations of chemotherapy drugs are given at the same time.

Another promising target for cancer targeted therapy is angiogenesis. Several drugs, including some naturally occurring compounds, have the ability to inhibit angiogenesis and two compounds in this class are angiostatin \& endostatin; both are derived from naturally occurring proteins. These drugs prevent angiogenesis happened by tumor cells, restricting tumor growth and preventing metastasis. Important advantage of angiogenesis inhibitors is that, there is less possibility that cancer cells will develop resistance to the drug because they do not target the cancer cells directly.

A class of drugs called spindle inhibitors (stop the synthesis of microtubules) stops cell replication early in mitosis. Experts have suggested that in order to overcome the side effects, efforts should be aimed at finding new and efficacious anticancer drugs based on identification of the cell cycle targets that interfere with the cell cycle regulatory pathway. [1.2]

Immunotherapy encompasses different techniques which use the immune system to attack/hit/target cancer cells or treat the side effects of several types of cancer treatments. The least specific of these are the immunostimulants, such as interleukin 2 and alpha interferon that stimulates the normal immune response. A technique called chemo immunotherapy that attaches chemotherapy drugs to antibodies, are specific for cancer cells. The antibody then delivers the drug directly to cancer cells without affecting normal cells, lowering the toxic side effects of chemotherapy. These molecules contain two parts: one, cancer-cell specific antibody, two, and the drug that is toxic once it is taken into the cancer cell.

A similar strategy, radio immunotherapy, couples specific antibodies to radioactive atoms, thereby targeting the deadly radiation specifically to cancer cells. On the other hand, another immunological process uses antibodies that inactivate cancer-specific proteins, such as growth factors or tumor cell receptors that are important to tumor cells (research done on breast \& ovarian cancer and drug discovered for receptor protein called HER2). [7]

\section{ROLE OF BIO-INFORMATICS}

Modern technological improvement of large scale expression analysis of gene is utilizing DNA micro-arrays and proteomics following the bio-informatics methods. Different efficient tools \& algorithms are developed to understand detailed shape of proteins \& biological molecules to design drug with more disease resistant capability. The area of cancer research is not an exception $[16,19,21]$.

\section{Protein Structure Determination}

Scientists are using the idea of bio-informatics in protein 3dimentional structure prediction which is better than traditional 2-D structure prediction. 3-D structure of a protein helps in protein classification, interaction with other macromolecules, prediction of functions for uncharacterized proteins, interactions with small ligands like metal ions, nucleotides, substrates, co-factors and inhibitors, enzyme mechanism, structure based drug development, understanding of different boding, predicting active site for targeted therapy like cancer, experimental evidence for transmembrane domains. Right now, only about 10 percent of known protein sequences structures are determined using experimental (costly, slow, and unusable for some proteins) or computational (more power) methods.

The Protein Data Bank which is a freely accessible database of 3-Dimentional protein structures, begun in 1971 with seven structures which has nearly 40000 structures now, with the yearly number of structures added to the database that increases each year. In 2004 and 2005 over 10000 new structures were added to the database. Recently only $3 \%$ were obtained using computational models from PDB.

\section{Cancer classification approaches}

Traditional Microarrays and serial analysis of gene expressions are two recent technologies for measuring the thousands of genome expression values in parallel. The first consists of c-DNA microarrays and high-density oligonucleotide arrays, quantifies the relative levels of mRNA abundance in between different samples, while the later measures the absolute level of abundance.

DNA microarray has been used by researchers to identify genes involved in metastasis. Researchers investigated the colonial relationship of 22 liver tumors from six patients through gene expression profiling by cDNA microarray containing of 23,000 genes. The study aimed at identifying metastasis genes for liver cancer. They were able to identify a total of 63 genes (out of which 39 are known genes and 24 are expressed sequence tags) up-regulated and 27 genes (out of which 14 known genes \& 13 are expressed sequence tags.) down-regulated in metastasis modules compared to primary tumors. There is no single classifier identified that is superior over the rest. Some of the methods only work well on binary class problems but not extensible to multi class problems, where others are more general and flexible. The thing is to note for most of those proposed algorithms on gene classification are the authors are only concerned with the exactness of the classification and did not pay much attention to the running time $[5,6,8]$.

Extensive study reveals below challenges with cancer classification problems:

- Available public gene expression data for cDNA microarrays is very small. (Colon tissue samples, the Leukemia data set, etc., has sample size below 100)

- The number of genes, of the data or the attribute space is enormous: there are generally thousands to hundred thousands of genes present in each tuple. If the samples are mapped to point in the attribute space, then the samples can be seen in a very high dimensional space as very sparse points. Most existing classification algorithms were not designed with such kind of data characteristics keeping in mind. Such a situation of sparseness and high dimension is a big challenge for most classification algorithms and over fitting is a major problem due to the high dimension; on the other hand the small data size makes it worse. Additionally, with so many genes in the tuple, it will be a big challenge on computation time. Hence, developing an effective and efficient classification algorithm for cancer classification is not an easy task.

- The presence of noise inherent in the data set (biological noise [introduced by genes that are not relevant for 
determination of the cancer classes \& are associated with the non-uniform genetic backgrounds of the samples or the misclassification of the samples] \& technical noise [are introduced at the various stages of data preparation]). The presence of noise makes accurate classification of data difficult, after coupled with small sample size.

- Dealing with a huge number of irrelevant attributes (genes). Most genes are not cancer related. The presence of these irrelevant genes, interferes with discrimination power of those relevant attributes. This not only incurs extra computation time with respect to training and testing phase of the classifier, but also increases the classification difficulty.

- Application domain of cancer classification. Both Accuracy \& Biological relevancy are important in cancer classification. Some useful information can be gained from the classification process is the determination of the genes that work as a group to determine cancerous cells or the genes that are underexpressed or over expressed in certain tissues or cells. All such would help biologists to gain more understanding about the genes and how they work together and interact with each other. So, biologists are more interested in classifiers that not only produce high classification accuracy but also exhibit important biological information.

\begin{tabular}{|c|c|c|c|c|}
\hline & $\begin{array}{c}\text { Nulti- } \\
\text { class }\end{array}$ & Strategy & $\begin{array}{c}\text { Biologically } \\
\text { meaningful }\end{array}$ & Scalability \\
\hline \hline SWM & No $_{0}$ & Max-Margin & $N_{0}$ & Good \\
\hline Boosting(Decision-tree) & Yes & Max-Margin & Yes & Classifier Dependent \\
\hline Decision tree & Yes & Entropy Function & Yes & Good \\
\hline KNN $(K \leq 1)$ & Yes & Similarity & No & Not Scalable \\
\hline CAST & Yes & Similarity & No & Not Scalable \\
\hline G'S & No & Weighted Voting & Yes (gene selection) & Fair \\
\hline FLDA & Yes & Discriminant Analysis & $N_{0}$ & Fair \\
\hline Neural Network & Yes & Perceptrons & $N_{0}$ & Fair \\
\hline Naire Bayes & Yes & Distribution modelling & No & Fair \\
\hline
\end{tabular}

Fig: 2 Summarization of Cancer classifiers

Publicly available cancer data sets for cDNA microarray:

- Colon cancer data: This data consists of 62 samples of colon epithelial cells obtained from colon-cancer patients. Samples consist of tumor biopsies collected from tumors, and normal biopsies obtained from healthy part of the colons of that patient. The number of genes in the data set is more than 2000 .

- Ovarian cancer data: It consists of 32 samples, 15 of which are ovary biopsies of ovarian carcinomas, 13 are biopsies of normal ovaries and remaining 4 samples belong to other tissues. 28 of the samples are labeled.

- Leukemia data: This data consists of 72 samples. The samples consist of two types of leukemia, 25 are AML and 47 are ALL. Samples are taken from 63 bone marrow samples and 9 peripheral blood samples. There are 7192 genes in the data set.

- Lymphoma data set: This data consists of 81 samples with 4,482 genes. In the 81 samples, 29 of them belong to class 1 , other 9 samples belong to class 2 and remaining 43 samples belong to class 3 .

- NCI data set: This data consists of 60 samples of 9,703 genes obtained from the National Cancer Institute (NCI)'s anti-cancer drug screen. The 60 samples belong to a variety of classes like 7 belongs to breast, 5 belongs to central nervous system, 7 belongs to the colon, 6 belongs to leukemia, 8 in melanoma, 9 in non-small-cell-lung carcinoma, 6 in ovarian class, 2 in prostate, 9 in renal and 1 of unknown. There is another data set from NCI. It consists of samples of 218 normal tissue and samples of 90 cancerous tissues spanning across 14 different cancer types. There are 16,063 genes in this data set.

\section{THERAPIES OF CANCER: PRESENT \& FUTURE}

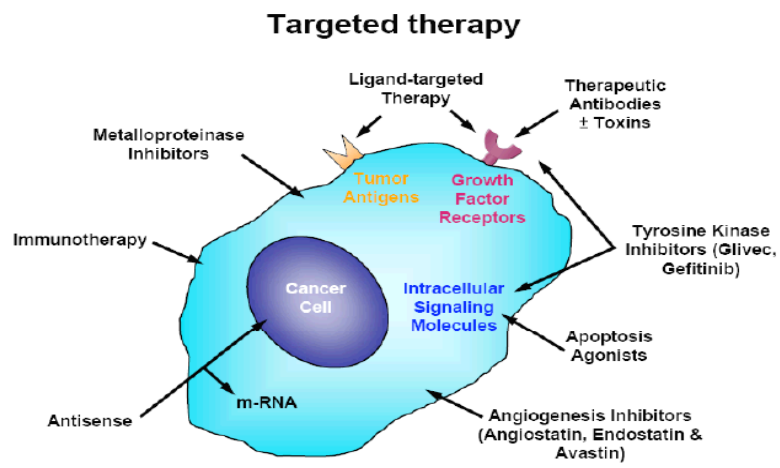

Fig: 3 Drug Target Classes

This paper includes present $\&$ future scope of peptide targeted therapies with other ongoing researches as follows:

- Peptide Targeted Therapy: There are certain limitations in the use of proteins, MoAbs or large protein ligands like poor delivery to affected cancer cells due to their large size $\&$ doselimiting toxicity to the liver $\&$ bone-marrow cancer.

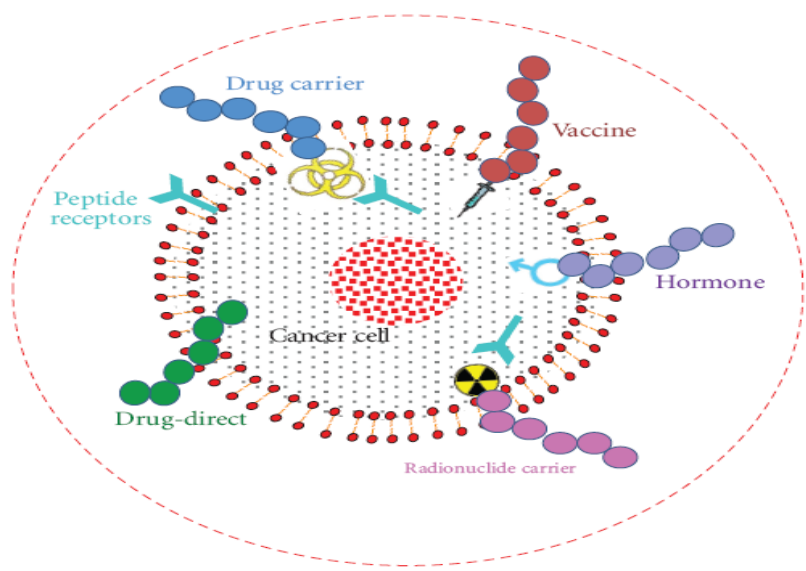

Fig: 4 Different treatment options with Peptides

Drugs like Enzyme inhibitors inhibit enzymes that signal for cancer cells to grow. Inhibition stops growing \& spreading of cancer cells. The tumor is not getting smaller means its abnormal growth has been interrupted. This may give normal chemo a better chance to work on. Slowing or stopping outof-control growth may also help people live longer, even without adding other drugs. The enzyme inhibitors are called by different names based on the enzymes they block like Tyrosine kinase inhibitors, mTOR inhibitors, Proteosome inhibitors, Growth factor inhibitors, Signal-transduction 
inhibitors, Multi-targeted kinase or multikinase inhibitor. Over 400 proteins used as drug targets. Sequence analysis of these proteins reveals that most targets fall within a few major gene families (GPCRs, kinases, proteases and peptidases).

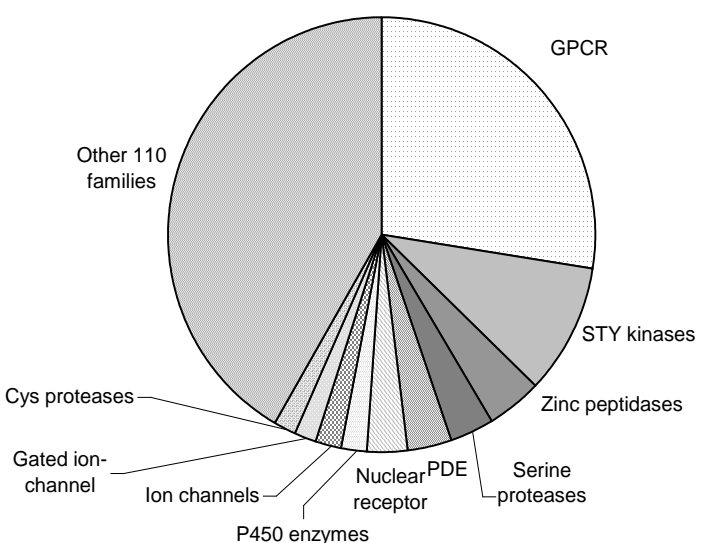

Fig: 5 Gene Families

Prime growth factor of normal cell is hormones. Although cancer cells have lost some of the normal responses to growth factors, but some cancer cells still require hormones for growth. Hormone therapy for cancer attempts to starve the cancer cells for these hormones. It is usually done with drugs that block the activity of the hormone, where some drugs can block synthesis of the hormones. Drugs block the binding site for hormone to slow the growth of these cancers. Research proves that it is effective for prostate \& breast cancer. $[1,2$, 5].

\section{Ongoing Researches \& findings:}

A new chemotherapy drug has been invented that will target the cells that are holding structure of cancer cell and will force self-destruction. The research has been carried out at University of New South Wales, Australia and its name is TR100. It has the capability to target that particular protein that forms the cancer cell structure. It does not attack healthy cells. Research has been carried out in lab on rats. TR100 understands \& destroys two changed tropomyosins which are used by cancer. It has been tested on melanoma and neuroblastoma.

Chemotherapy brings a serious toll on cancer patients. It can temporarily or permanently affect fertility. A new invention has been carried out at Northwestern University's Feinberg School of Medicine where a new chemotherapy drug has been proposed that can do both preserve fertility and destroys cancer. It is also a target based therapy where nanoparticle chemo drug (arsenic trioxide) targets cancer cells. It does not affect ovarian tissue, follicles, and eggs as per the ongoing research.

More than 30000 cases has been reported on acute myeloid leukemia in USA, Europe \& ASIA. AML is very much popular among adults and very aggressive in nature where survival rate is 40 to 45 percent with age less than sixty and 10 percent with age more than sixty. Research has been carried out at Research Center for Allergy and Immunology in Japan and found a bio-inhibitor for leukemia stem cells which survive chemotherapy due to the presence of hematopoietic cell kinase \& can cause an AML relapse. Researchers have uncovered a compound namely RK-20449 that blocks HCK. so, stem cell has become a new battle field of Leukemia treatment.

Large scale of study has been carried out on about 23,000 women begun in 1988 and found that $15 \%$ of breast cancer patients are less likely to die who drank alcohol before they started diagnosis, $25 \%$ of breast cancer patients are less likely to die who drank alcohol after they started breast cancer diagnosis, from cardiovascular conditions, 39\%-50\% of breast cancer patients are less likely to die who drank alcohol before or after they started breast cancer diagnosis, from cardiovascular conditions.

Research at University of Montreal Hospital Research Centre (CRCHUM) in Canada has been carried out on a bio-gel that contains functional $\mathrm{T}$ lymphocytes and can destroy the cancerous cells. The gel is non-toxic and $\mathrm{T}$ lymphocytes within it can grow for 2-3 weeks and fight. It can be injected directly into cancer tumor rather in the bloodstream. It has been successfully tested on melanoma and kidney cancer in lab.

A dosage of $75 \mathrm{mg} /$ day or greater of daily aspirin consumption, reduces death rates by cancer as suggested by study.

Latest study shows around 93K new cases are reported in USA each year in colorectal cancer. Research has been found on test lab that high concentration of vitamin-C (equivalent to 300 oranges) weakens the growth of KRAS and BRAF mutant colorectal tumors.

The study shows that daily consumption of 50gram of processed meats can increase the chance of colorectal cancer by $17 \%-18 \%$ and around $34 \mathrm{~K}-35 \mathrm{~K}$ annual deaths. High intake of red meats can lead to pancreatic and prostate cancers.

Latest research activity in University of Chicago on melanoma, has been performed into gut of mice which has shown a drastic anti-cancer affects. Cancer cells basically destroy our T-cells and checkpoint inhibitors like anti-PD-L1 antibodies, restricts the tumor cells to get attached to the $T$ cells. Addition of some bacterial flora in the digestive tract of mice has drastically increased immunity level to fight against tumor cell and they behave like checkpoint inhibitors.

Study at Dana-Farber Cancer Institute has shown that colorectal cancer has a relation with immune system. High level of vitamin-D makes cells more resistant and improves immune system. Experiment was carried out on 942 people.

Biomedical engineers have developed a lab enhanced natural killer cell with the help of nanotechnology that are capable to eliminate cancer in lymph nodes. We all know that lymph node metastasis is a serious problem and using the natural killer injection, cancer spreading can be stopped by lymph nodes. Successful result has been obtained from mice and Prof. King and colleagues have found a way to boost the capability of the killer by attaching a protein called TRAIL to make it "super killer cell".

The research as per Proceedings of the National Academy of Sciences, scientists have found that there is a way to stop spreading of cancer infected cells through bloodstream by simply self-destroying mechanism. Radiation/Surgery are good for cancers when they are in early stage but when they are one the way to spread then the sitation becomes worse! Cancer cells destroy themselves when they come in the 
contact of Trail coated WBC. In lab, the destruction rate has been found $100 \%$ in a flowing model of human blood stream.

Latest treatments have shown a path that removal of lymphs are not required while treating breast cancer as it may cause many unwanted side affects!

Misguiding lymphoma cells and followed by increasing immune responses is a new therapy in Lymphomas. Natural killer cells or NK cells act as soldier to fight against viral infection and cancers. Research proves that NK cells have the ability to attack B-lymphoma cells. Lymphoma cells can destroy NK cells either by a cytokine called IL-10 or by deactivating NKG2D ligands. NK cells resist against Blymphoma in earlier stage by producing cytokine called interferon-gamma. According to the therapeutic approach, injecting IFN- $\gamma$ or antibodies against IL-10 can produce resistance against lymphoma.

Researchers at MIT and UCSD have proposed a new idea to detect cancer by genetically modified beneficial bacteria.

Observation has been found after injecting E.Coli into mice, engineers were able to detect liver cancer.

Research has been carried out with liposomes to use it as a passive target. Due to retention effect \& permeability, give passage to larger molecules. So, drugs encapsulated with liposomes with size of $400 \mathrm{~nm}$ can easily enter tumor site. But, they are prohibited to enter in healthy cell site by their endothelial wall. Anthracyclines is a class of anti-cancer drug is used for the same.

Research is going on fruit flies in cancer research by Geneticist.

Recent research has unfolded a fact that capsaicin found in chillies and responsible for fiery hot taste have anticancer properties.

Strange observation on African children by Dr Robbiani malaria is affecting immune system and sometime causing Burkitt's lymphoma.

\section{MATHEMATICAL MODELING \& APPROACHES}

The Interest in human cancer has given birth to a new multidisciplinary approach that synthesizes biological data \& hypothesis with mathematical modeling. It is emerging mathematical approaches are important to set up a coherent framework to make out the complex intra \& extra cellular dynamics that drives tumor biology.

A good model is like a 'Virtual Laboratory' where system parameters \& hypothesis can be varied systematically \& tested in the ways that would not be feasible experimentally.

Some mathematical modeling on the top of bio-informatics:

- Multiscale modeling (based on tumor growth).

- Tumor vascular modeling (based on angiogenesis in tumor biology \& can be used to investigate drug discovery protocol along with exploring the effect of heterogeneity of oxygen concentration on the growth dynamics of cells).

- Population models (used for tumor behavior formalization on the concept of carcinogenesis as somatic evolution). [20].

\section{FOOD HABIT}

Correct food habits \& required food charts are shown below from different sources to avoid cancers as much as possible:

\begin{tabular}{|c|c|}
\hline Don't & Dós \\
\hline Traditional Western diet & Mediterranean, Indian and Asian cuisine \\
\hline White bread and pasta & Whole-wheat bread and pasta \\
\hline Red meat, raised industrially & $\begin{array}{l}\text { Organic meat from animals fed on grasss or with } \\
\text { flax meal, eaten at most three times a week }\end{array}$ \\
\hline $\begin{array}{l}\text { Oils rich in omega-6fatty ycids (corn, sunflower, } \\
\text { safflower, soy) }\end{array}$ & Oliveoil \\
\hline $\begin{array}{c}\text { Dairy products from industrialy rajised livestock } \\
\text { (especialy full fatt) }\end{array}$ & Dairy products mainly from animals fed on grass \\
\hline $\begin{array}{l}\text { Egggfrom industrial farming hens fed corn and soy } \\
\text { beans) }\end{array}$ & $\begin{array}{c}\text { Eggs of hens rajesed in a natural environmentorfed } \\
\text { flax meal }\end{array}$ \\
\hline Unmanageed stress, anger and depression & Laughter, lightheartedness, serenity \\
\hline Less than 20 minutes of physical activity a day & $\begin{array}{l}\text { A50-minute wakk three times a week or } 30 \\
\text { minutes six times a week }\end{array}$ \\
\hline $\begin{array}{l}\text { Cigarette smoke, atmospheric pollution, domestic } \\
\text { pollutants }\end{array}$ & Clean environment \\
\hline
\end{tabular}

Fig: 5 Proper Habits

\begin{tabular}{|c|c|}
\hline Foods & Reason to refrain from \\
\hline Soda Pop & derivative 4-methylimidazole (4-MI) \\
\hline Farmed Salmon & PCB's, mercury, and cancer causing dioxins \\
\hline Processed Meats & chemicals and preservatives, including sodium nitrates \\
\hline Smoking Meats & Tar \\
\hline Microwave Popcorn & perfluorooctanoic acid (PFOA) \\
\hline Potato Chips & acryl amide \\
\hline Hydrogenated oils & excess of Omega- 6 fatty acids \\
\hline Foods that are highly salted, pickled, or \\
smoked
\end{tabular}




\begin{tabular}{|c|c|}
\hline Foods & Reason to take \\
\hline $\begin{array}{l}\text { Antioxidants - } A \text { substance, such as vitamin } E \text {, } \\
\text { vitamin } C \text {, or beta carotene }\end{array}$ & $\begin{array}{l}\text { to protect body cells from the damaging effects } \\
\text { of oxidation }\end{array}$ \\
\hline $\begin{array}{l}\text { Phytochemicals-A nonnutritive bioactive plant } \\
\text { substance, such as a flavonoid or carotenoid }\end{array}$ & $\begin{array}{l}\text { considered to have a beneficial effect on human } \\
\text { health }\end{array}$ \\
\hline $\begin{array}{c}\text { Curcumin (turmeric), Paprika, Carotenoids, } \\
\text { Lycopene, Sulforaphane, Flavonoids, Zeaxanthin }\end{array}$ & $\begin{array}{c}\text { considered to have a beneficial effect on human } \\
\text { health }\end{array}$ \\
\hline $\begin{array}{l}\text { Beets, GREEN TEA (Anti-oxidant),TURMERIC, } \\
\text { GARLIC, ONIONS, LEEKS, SHALLOTS and CHIVES, } \\
\text { MUSHROOMS (Shiitake, crimini, portabello and } \\
\text { oyster mushrooms), CRUCIFEROUS VEGETABLES } \\
\text { (Cabbages, sprouts, broccoli and cauliflower), } \\
\text { FRUITS AND VEGETABLES RICH IN CAROTENOIDS } \\
\text { (Carrots, yams, sweet potatoes, squash, tomatoes, } \\
\text { apricots, beets and all the brightly colored fruits and } \\
\text { vegetables contain vitamin A and lycopene), HERBS } \\
\text { AND SPICES (Rosemary, thyme, oregano, basil and } \\
\text { mint), CITRUS FRUIT(Oranges, tangerines, lemons } \\
\text { and grapefruit contain anti-inflammatory } \\
\text { flavonoids) }\end{array}$ & $\begin{array}{l}\text { Gives immunity, acts as cancer enhancing } \\
\text { enzyme blocker, protects breast, liver, lung, } \\
\text { prostate, skin, stomach, and bladder cancers }\end{array}$ \\
\hline Berries & $\begin{array}{c}\text { Protects colon, esophageal, oral, and skin } \\
\text { cancers as contains Phytochemicals called } \\
\text { anthocyanins }\end{array}$ \\
\hline Tomatoes & $\begin{array}{l}\text { Protects endometrial, lung, prostate, and } \\
\text { stomach cancers due to presence of lycopene }\end{array}$ \\
\hline Walnuts & $\begin{array}{l}\text { Protects breast and prostate cancers as } \\
\text { phytosterols is present and it blocks estrogen } \\
\text { receptors in breast cancer }\end{array}$ \\
\hline Garlic & $\begin{array}{l}\text { Protects breast, colon, esophageal, and stomach } \\
\text { cancers and halts the formation of nitrosamines, } \\
\text { carcinogens formed in the stomach }\end{array}$ \\
\hline Beans & $\begin{array}{l}\text { Protects breast and colon cancers as fatty acid } \\
\text { butyrate is present }\end{array}$ \\
\hline
\end{tabular}

Fig: 7 Required Food Chart

\section{CONCLUSION}

As per the report of American cancer society \& WHO, the time has come to fight back as the war has begun. The death rate is increasing rapidly as time passes.

The diagnosis at earlier stage increases the chance of survival so test for cancer should be conducted on regular basis. Regular genetic tests \& cancer risk counseling is required if any family has strong cancer history. Genomic technology has made drug target analysis effective with the help of bioinformatics.

Targeted therapies are being considered to be the future of cancer treatments \& many researchers are going on to produce successful result. Different Bio-informatics tools are also being used in the research to have some stable drug design along with evolutionary algorithms.

Many new molecular targeted cancer drugs have gained approval over the last few years and have improved and extended the lives of a large number of patients. However, the discovery and development of new targeted drugs have some drawbacks like it is still frustratingly slow and have high failure rates, mainly in late stage clinical trials leading to a constant challenge.

Our understanding of the genetics and molecular basis of cancer initiation and malignant advancement has improved enormously, with the help of bio-informatics. It has opened up astounding possibilities for selective therapeutic targeting to exploit addiction, dependency and vulnerabilities in cancer cells. Scientific and technological breakthroughs have enabled faster efficient drug discovery, together with more refined clinical trials.

Progress has been made in large scale genetic and molecular characterization of cancer to accelerate bringing new personalized drugs to cancer patients worldwide. Systematic and impartial formulations to cancer classification are of great importance to cancer treatment and drug discovery. Former cancer classification methods were limited in their diagnostic ability due to they all were clinical based. It has been known that gene expressions incorporate the keys to the primal problems of cancer diagnosis, treatment and drug discovery.

The recent arrival of microarray technology has made the production of huge amount of gene expression data possible. This helps the researchers to propose different cancer classification algorithms using gene expression data.

The link between cell cycle \& chromatin control has led to open up the mechanisms to explain abnormalities of tumor cells that can be targeted to design new drugs. Targeting chromatin control may yield valuable information.

Latest research has revealed that cancer is less expressed in elephants than human as elephants inherit 20 copies of TP53/Tumor suppressor genes whereas human inherits only 2 copies from each parent. If in human that count becomes less than 2 then there is a chance of cancer. A new drug on neuro $\&$ endocrine cancers has been launched at a conference few days back organized by Amrita Center for nano-sciences, headed by Manzoor Koyakutty.

Along with medical advancement, our lifestyle \& food habit must be under control to fight against this disease.

\section{REFERENCES}

[1] Gibbs, W. Wayt. 2003. Untangling the roots of cancer. Scientific American, July, 57-65. New evidence challenges old theories of how cancer develops.

[2] Han-Chung Wu1, De-Kuan Chang, and Chia-Ting Huang. Journal of Cancer Molecules 2(2): 57-66, 2006. Targeted Therapy for Cancer.

[3] Harris M. Monoclonal antibodies as therapeutic agents for cancer. Lancet Oncol 5: 292-302, 2004.

[4] Wilson, J. F. 2001. A dual role for CDK inhibitors. The Scientist 16[6]:20. Discusses approaches to cancer treatment using cells' cycle inhibitors.

[5] G Roti and K Stegmaier. British Journal of Cancer (2012) 106, 254 - 261. Genetic and proteomic approaches to identify cancer drug targets

[6] Chung CH, Levy S, Chaurand P, Carbone DP. 2007. Genomics and proteomics: Emerging technologies in clinical cancer research. Crit Rev Oncol Hematol 61:125 .

[7] Galvão ER, Martins LM, Ibiapina JO, Andrade HM, Monte SJ. 2011. Breast cancer proteomics: a review for clinicians. J Cancer Res Clin Oncol. 137(6):915-25.

[8] Kihara D, Yang YD, Hawkins T. 2007. Bioinformatics resources for cancer research with an emphasis on gene function and structure prediction tools. Cancer Inform 7;2:25-35. 
[9] Swen Hoelder, Paul A. Clarke, Paul Workman. MOLECULAR ONCOLOGY 6 (2012) 155-176. Discovery of small molecule cancer drugs: Successes, challenges and opportunities.

[10] Veggeberg, S. 2002. Fighting cancer with angiogenesis inhibitors. The Scientist 16[11]:41. Discussion of a class of drugs that helps to prevent angiogenesis.

[11] Dvorak HF, Nagy JA, Dvorak AM. Structure of solid tumors and their vasculature: implications for therapy with monoclonal antibodies. Cancer Cell 3: 77-85, 1991.

[12] Shockley TR, Lin K, Nagy JA, Tompkins RG, Dvorak HF, Yarmush ML. Penetration of tumor tissue by antibodies and other immunoproteins. Ann N Y Acad Sci 618: 367-382, 1991.

[13] Alicia S. Chung, John Lee \& Napoleone Ferrara. Nature Reviews Cancer 10, 505-514 (July 2010) doi:10.1038/nrc2868. Targeting the tumor vasculature: insights from physiological angiogenesis.

[14] Shanju Sankar, Sangeetha K Nayanar, Satheesan Balasubramanian. Asian Pac J Cancer Prev, 14 (7), 40414047.

DOI:http://dx.doi.org/10.7314/APJCP.2013.14.7.4041. current Trends in Cancer Vaccine - a Bioinformatics Perspective.
[15] Christoph Bock and Thomas Lengauer. Vol. 24 no. 1 2008, pages 1-10.doi:10.1093/bioinformatics/btm546. Computational epigenetics.

[16] Thomas Lengauer and Ralf Zimmer. Henry Stewart Publications 1467-5463. BRIEFING IN BIOINFORMATICS. Vol 1,No 3, 275-288, September 2000. Protein structure prediction methods for drug design.

[17] Azuaje. Interpretation of genome expression patterns: computational challenges and opportu-nities. IEEE Engineering in Medicine and Biology, 2000.

[18] Berns. Cancer: Gene expression in diagnosis. Nature, pages 491-492, Feb 2000.

[19] Wang XD, Liotta L. 2011. Clinical bioinformatics: a new emerging science. J Clin Bioinforma 2011, 1(1):1.

[20] Ayan Chatterjee \& Dr. Uttam Kumar Roy, International Journal of Current Engineering and Technology 12/2015; 5(6):3866. DOI: 10.14741/Ijcet/22774106/5.6.2015.66

[21] Ayan Chatterjee \& Dr. Uttam Kumar Roy, International Journal of Scientific and Engineering Research 11/2015; 6(11):612. DOI: 10.14299/ijser.2015.11.006 